\section{Antitranspirante mit Aluminiumchlorid}

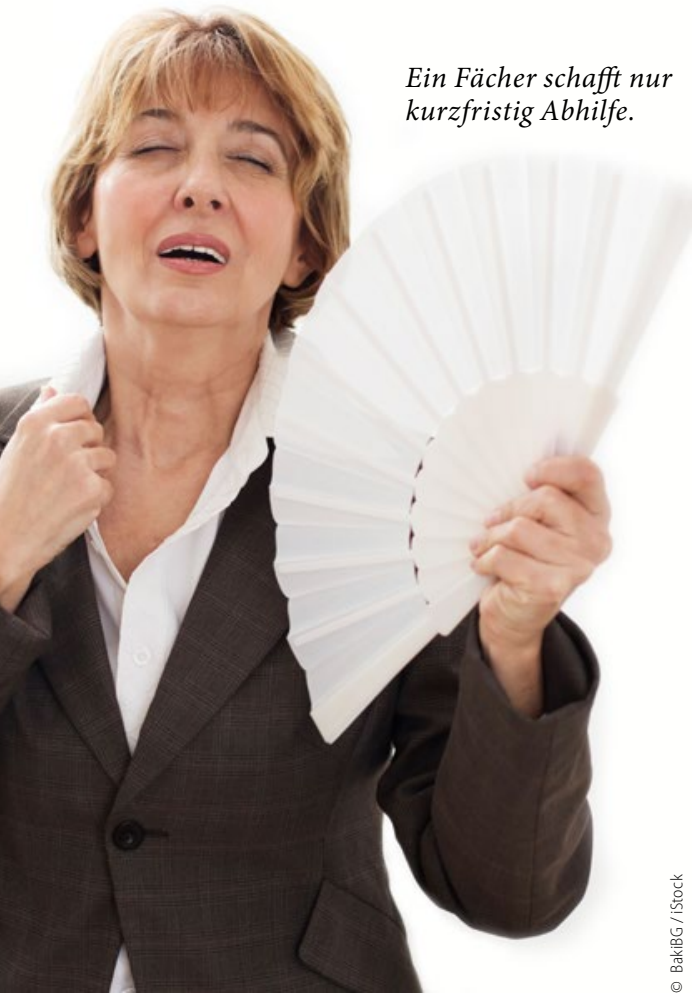

Zellulose die Verträglichkeit des Antitranspirants.

Anders als Deodorants und ein herkömmliches Antitranspirant muss Sweat-off nicht täglich oder mehrmals am Tag aufgetragen werden. Es genügt eine kurze „Eingewöhnungsphase“ von drei bis vier Anwendungen. Anschließend genügt eine Wiederholung nur noch bei Bedarf wenn es nötig ist zum Beispiel nach ein bis zwei Wochen.

Der Sweat-off Roll-on mit $50 \mathrm{ml}$ Inhalt hält je nach Anwendung bis zu einem Jahr. Das Antitranspirant ist ohne Alkohol, Farbstoffe, Parfüm und Konservierungsmittel und eignet sich für Männer und Frauen gleichermaßen. Wie bei allen Sweat-off-Antitranspiranten wird der Roll-on abends vor dem Schlafengehen hauchdünn auf die saubere, trockene und intakte Haut auftragen.

Das Sweat-off zero Deodorant wurde ergänzend zur täglichen Anwendung gegen Schweißgeruch entwickelt. Das Deodorant wirkt sehr gut antibakteriell und neutralisiert geruchsbildende Bakterien und dadurch die Ursache des störenden Körpergeruchs.

Nach Informationen von Sweat-off
Onychomykose

\section{Topische Behandlung mit medizinischem Nagellack}

Erstmals wurde die Wirkung von antimykotischen Nagellacken mit den Wirkstoffen Ciclopirox $8 \%$ (Prüfpräparat: Ciclopoli ${ }^{\oplus}$ gegen Nagelpilz) und Amorolfin $5 \%$ (Prüfpräparat: Loceryl ${ }^{\circledR}$ Nagellack) direkt verglichen [Iorizzo $M$ et al. Skin Appendage Disord 2015;1:134-40]. 120 Patienten mit milder bis moderater Onychomykose des großen Zehennagels wurden in zwei Gruppen aufgeteilt und über 48 Wochen hinweg behandelt; die Auswertung erfolgte verblindet. Die eine Gruppe trug täglich Ciclopirox auf; die andere Gruppe wendete Amorolfin zweimal wöchentlich an.

Als primärer Endpunkt der Studie war die Umwandlung zur negativen mykologischen Kultur nach 12 Wochen definiert. Dieser Endpunkt wurde erreicht; eine Nicht-Unterlegenheit von Ciclopirox gegenüber Amorolfin wurde festgestellt. Weiterhin weisen die Untersuchungsdaten im sekundären Endpunkt nach 48 Wochen auf eine statistisch signifikante überlegene Wirksamkeit von Ciclopirox versus Amorolfin hin: Der Behandlungserfolg, definiert als $\geq 90 \%$ gesunder Nagel und $100 \%$ mykologische Heilung, betrug bei Ciclopirox 58,3\%; im Falle von Amorolfin 26,7 \%.

Nach Informationen von Taurus Pharma
Aufhellende Wirkung für gleichmäßigen Teint

\section{Schutz und Melaninregulierung bei bestehenden Pigmentflecken}

FotoUltra Active Unify von ISDIN setzt mit seinem DP3Unify-Komplex direkt am Hauptmechanismus der Melanogenese an und sorgt so für eine aufhellende Wirkung sowie einen gleichmäßigen Teint.

Auch Studien haben die starke Wirksamkeit unter Beweis gestellt: nach vier Wochen konnte bei $70 \%$ der Patienten eine pigmentreduzierende Wirkung nachgewiesen werden [Gomes-Neto et al. Efficacy of new topical formulation in melasma patients. EADV 2013]. Der sehr hohe Lichtschutzfaktor (UV-B 119, UV-A 49) bietet zweimal höheren Schutz vor UV-A-Strahlen als mindestens erforderlich bei LSF 50+. Durch die innovative Fluid Textur zieht der Wirkstoff schnell ein und macht so die tägliche Anwendung einfach und angenehm.

FotoUltra Active Unify ist eines von drei medizinischen Sonnenschutzprodukten der Firma ISDIN: FotoUltra Solar Allergy zur Linderung der Symptome einer Sonnenallergie sowie FotoUltra SporPrevent zur Prävention von Pigmentveränderungen runden das Produktportfolio ab. 